\title{
Tissue engineering and regenerative medicine research - how can it contribute to fight future pandemics?
}

\author{
https://doi.org/10.21814/uminho.ed.24.18
}

\section{David Caballero}

David Caballero (ORCID: 0000-0001-7930-2535) is a biophysicist with an M.Sc. and a Ph.D. in Nanoscience from the University of Barcelona (Barcelona, Spain). Currently, he is a research associate at the I3Bs (Research Institute on Biomaterials, Biodegradables and Biomimetics of University of Minho) and ICVS/3B's (PT Government Associate Laboratory), working in the field of 3D tumor - and organ-on-chip based models for the study of tumor physiopathology.

\section{Mariana R. Carvalho}

Mariana R. Carvalho (ORCID: 0000-0003-2081-3909) has a Pharmacy background and $\mathrm{PhD}$ in the field of Tissue Engineering, Regenerative Medicine and Stem cells at the I3Bs (Research Institute on Biomaterials, Biodegradables and Biomimetics of University of Minho) and ICVS/3B's (PT Government Associate Laboratory). She is a postdoctoral fellow working in Tissue Engineered In Vitro Models on a Chip for Cancer Research under the project IET Harvey Research Prize 2017.

\section{Subhas C. Kundu}

Subhas C. Kundu (ORCID: 0000-0002-7170-2291) received his PhD in Genetics from Banaras Hindu University (India). His area of interest includes biomaterials for 3D cancer modeling and drug screening. Currently, he is an ERA Chair professor at the 3B's Research Group (I3Bs - Research Institute on Biomaterials, Biodegradables and Biomimetics of University of Minho and ICVS/3B's - PT Government Associate Laboratory of University of Minho). There he leads a multidisciplinary team fully dedicated in the development of a new generation of in vitro 3D tumor models for drug screening. 


\section{Joaquim M. Oliveira}

Joaquim M. Oliveira (ORCID: 0000-0001-7052-8837) is a biochemist and principal investigator at the I3Bs (Research Institute on Biomaterials, Biodegradables and Biomimetics of University of Minho) hired under the Prestigious Investigator FCT 2015 Program. Over the years, he has focused his work on the field of tissue engineering, nanomedicine, stem cells, and cells/drug delivery. More recently, he set up a new research line at the ICVS/3B's (PT Government Associate Laboratory of University of Minho) on $3 \mathrm{D}$ in vitro models of disease.

\section{Natália M. Alves}

Natália M. Alves (ORCID: 0000-0002-8741-4091) owns a PhD in Science and Engineering of Polymers by the School of Engineering of the University of Minho and is an Assistant Professor \& Research Staff member at the I3Bs (Research Institute on Biomaterials, Biodegradables and Biomimetics of University of Minho and ICVS/3B's - PT Government Associate Laboratory of University of Minho). She has been developing activities mainly related with smart and biomimetic polymers and biodegradable polymers from marine origin for biomedical applications.

\section{Rui L. Reis}

Rui Luis Reis, (ORCID: 0000-0002-4295-6129) PhD, DSc, Hon. Causa MD, Hon Causa PhD, FBSE, FTERM, member of NAE, FAIMBE, FEAMBES, is the Director of the 3B's Research Group (I3Bs - Research Institute on Biomaterials, Biodegradables and Biomimetics of University of Minho) and of the ICVS/3B's - PT Government Associate Laboratory of University of Minho). He is also CEO of the European Institute of Excellence on Tissue Engineering and Regenerative Medicine, Coordinator of the Discoveries Centre for Regenerative and Precision Medicine, Global Past-President of the Tissue Engineering and Regenerative Medicine International Society and Editor-in-chief of the Journal of Tissue Engineering and Regenerative Medicine. He is a recognized world expert in the TERM and biomaterials fields. 


\section{ABSTRACT}

Understanding the pathogenesis of viral infection is of paramount importance for the development of better therapies. In the particular case of COVID-19, the mechanism of infection is highly complex and involves a critical cascade of events, which can lead to the death of the patient. Intense research is currently being performed to gain mechanistic insights about the virus etiology and to evaluate new therapeutic approaches. The development of point-of-care diagnostic tools, predictive drug screening platforms, and biomimetic models of the disease could play a key role in understanding the cellular and molecular mechanism of viral infection and its response to drugs. In this regard, specific tissue engineering and regenerative medicine approaches, such as microfluidics and organ-on-a-chip technologies, as well as bio printed in vitro disease models, could be used to develop a technological platform to fight COVID-19, and other virus pandemics yet to come. Herein, we briefly discuss about how such approaches can contribute to address current and future viral pandemics by highlighting recent successful examples.

\section{INTRODUCTION}

Virus infections continue to be a major health problem worldwide. During the last decade, three main viral outbreaks have affected the population: MERS, SARS$\mathrm{CoV}$, and SARS-CoV-2. The latter virus causes the well-known COVID-19 disease, which was declared as world pandemics in March 2020 by the World Health Organization. This type of viral infections causes great expenditures to the governments and their national health systems. It is therefore vital to invest in groundbreaking technologies to gain mechanistic insights about the pathophysiology of the disease, which can lead to the development of better therapeutic solutions. In the particular case of COVID-19, solid evidences have shown that the infection is initiated when the SARS$\mathrm{CoV}-2$ virus enters the lung. Therein, the spike $(\mathrm{S})$ viral protein $(\mathrm{N}$-terminal portion of the viral protein unit $\mathrm{S} 1$ ) specifically binds to the angiotensin-converting enzyme 2 (ACE-2) receptor present on type II lung pneumocytes [1]. After infection, the virus is replicated initiating a critical cascade of events that, in severe cases, results in the death of the patient. The main two complications in COVID-19 responsible of this 
dramatic end are: (i) severe pneumonitis, which causes the asphyxia of the patient and (ii) massive blot clotting, which can lead to serious thromboembolisms. Both of them are life-threatening situations, and therefore, their rapid diagnostics is vital for a better patient prognosis. However, one of the main problems with COVID-19, and other viral diseases, is how to address the fatal cytokine storm that involves multiple organs and tissues, such as the lung, the heart, the kidney, and the vasculature. Indeed, there are no well-defined treatments for this type of patients as the actuation guidelines are evolving in a daily basis.

Current methods to investigate the etiology of pathologies, including viral infections such as COVID-19, and their response to medication are obsolete. Typically, pharmaceutical and biotechnological companies use traditional in vitro and in vivo animal - models to test drugs (Figure 1 A-B). The formers are based on standard tissue culture systems, such as Petri dishes or flasks. These methods have several advantages, such as high-throughput properties, low cost, or easy manipulation. However, they are also associated with serious drawbacks: cells are cultured in an artificial - flat - environment, and therefore, they show perturbed levels of gene expression. As a result, cells respond to drugs differently as they would do in the human body. In contrast, animal models show the needed biological, structural and rheological complexity similar to the native scenario. However, as widely acknowledged, these models are not predictive of the outcome of drugs in humans. In addition, they lack the human immune system, are critically controversial, and extremely expensive. Altogether, this limits our capacity to investigate the etiology of diseases. And importantly, this also makes that $80-90 \%$ of the drugs, which successfully reach the preclinical assay stage, to fail when tested in patients [2]. Therefore, there is the need for new point-of-care technologies and versatile solutions that are clinically relevant and capable to improve our understanding on disease etiology, its diagnosis, and in the development and screening of drugs. In particular, these new technologies should be capable to: (i) describe the physiopathology of the disease while being at the same time compatible with standard laboratory technologies; (ii) mimic the native (human) scenario and (iii) be cost-effective, portable, high-throughput, clinically-relevant, highly-sensitive, and mass produced. 


\section{Microfluidics}

During the last decade, a new generation of point-of-care technologies have emerged for the development of biomimetic in vitro models of human organs/tissues and its diseases. These models are based on microfluidics technology, and bridge the large gap between the afore-mentioned traditional in vitro systems and complex animal models. Microfluidics is defined as "the science and technology of systems that process or manipulate small volumes $\left(10^{-9}\right.$ to $10^{18}$ liters) of fluids, using channels with dimensions of tens to bundreds of micrometers" [3]. These channels are engrafted into "chips" of few centimeters in size, and are typically made of soft, biocompatible, and transparent materials (e.g., elastomers or hydrogels) (see Figure 1C). The channels are continuously perfused with fluid, such as culture media, plasma or whole blood, and recapitulate the architectures, physicochemical environments and interfaces on the native scenario.

Working with microfluidic devices is associated with several advantages compared to traditional in vitro and in vivo systems. This includes an enhanced speed and control over the different parameters, a reduced consumption of samples and reagents, capability of parallelization, the possibility to integrate sensors (and other electric components), or a higher efficiency, among others. In addition, the fabrication of microfluidic devices employs the technology developed for the microelectronics industry, making their fabrication cost very low. Briefly, the fabrication of microfluidic devices uses UV photolithography, chemical etching, and soft lithography methods to replicate a design from a mold using a biocompatible elastomeric material, such as polydimethylsiloxane (PDMS). Other more sophisticated techniques, such as 3D printing, micromachining, xurography, or injection molding can also be used. In this case, other (bio)materials are employed, such as hydrogels (e.g., collagen, gelatin, acrylamide-bisacrylamide, etc.) or polymers (e.g., polymethylmethacrylate, polystyrene, polycarbonate, etc.). The obtained resolution depends on the selected fabrication methods, but it is possible to fabricate structures from few $m m$ to sub- $m m$ resolutions. 

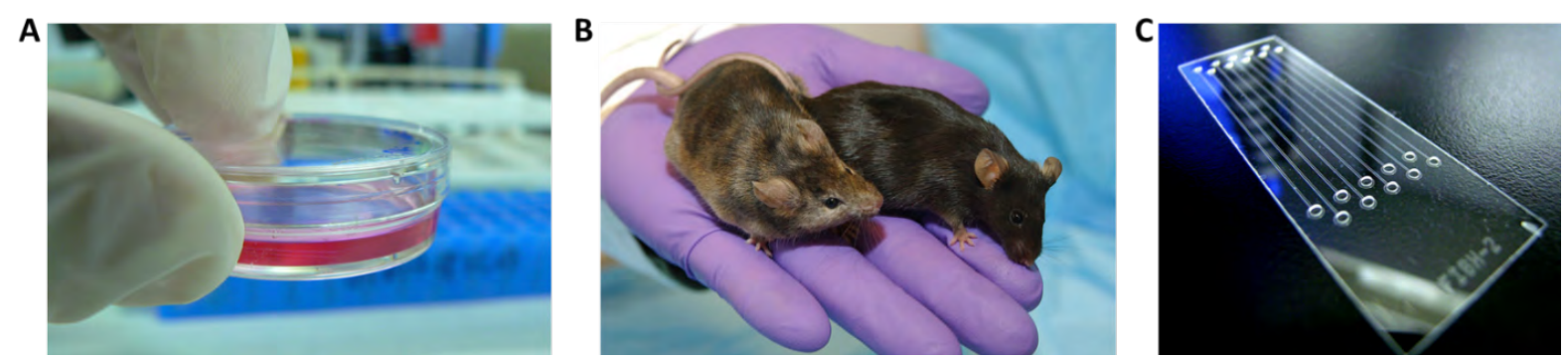

Figure 1. Systems for disease modelling and drug screening. (A) In vitro models; (B) In vivo - animal models; (C) Microfluidics device. (Image permissions: (A) Creative Commons Attribution 2.0 Generic; (B) National Institute of Health (US; Public domain); (C) Creative Commons Attribution 3.0 Unported).

The integration of human cells within microfluidic devices reproducing the functional units of human organs or tissues results into organ-on-a-chip systems. These advanced microfluidic models recapitulate the properties of the native tissue or organ (e.g., cell content, extracellular matrix, mechanochemical properties, etc.) and dynamic functions (e.g., biochemical gradients, fluid flow, shear stress, etc.). In this sense, organs-on-a-chip are considered as physiologically-relevant in vitro model platforms to study physio pathological processes, such as viral infections. They can also be utilized as powerful predictors of disease progression, drug efficacy, or for the identification of therapeutic targets or new disease biomarkers. Finally, the potential of organs-on-achip devices as screening platforms is supported by the number of companies that have emerged during the last years dedicated to the development of this type of technology [4]. Altogether, the advanced capabilities of microfluidic and organ-on-a-chip technology open unprecedented opportunities to gain key insights about the mechanism of virus infection and for assessing the efficacy of anti-viral drugs. Next, we will illustrate how microfluidics technology can contribute in managing the current and future virus outbreaks.

\section{HOW CAN MICROFLUIDICS TECHNOLOGY HELP IN VIRUS PAN- DEMICS?}

Microfluidics can significantly contribute in the current and future virus pandemic in three main scenarios: (i) by developing rapid and highly sensitive diagnosis 
tools of viral infection; (ii) by improving the efficiency of anti-viral drug discovery and screening; and (iii) by developing clinically-relevant disease models to investigate the etiology of virus infection (Figure 2). In the following, we briefly describe these three different types of contributions.

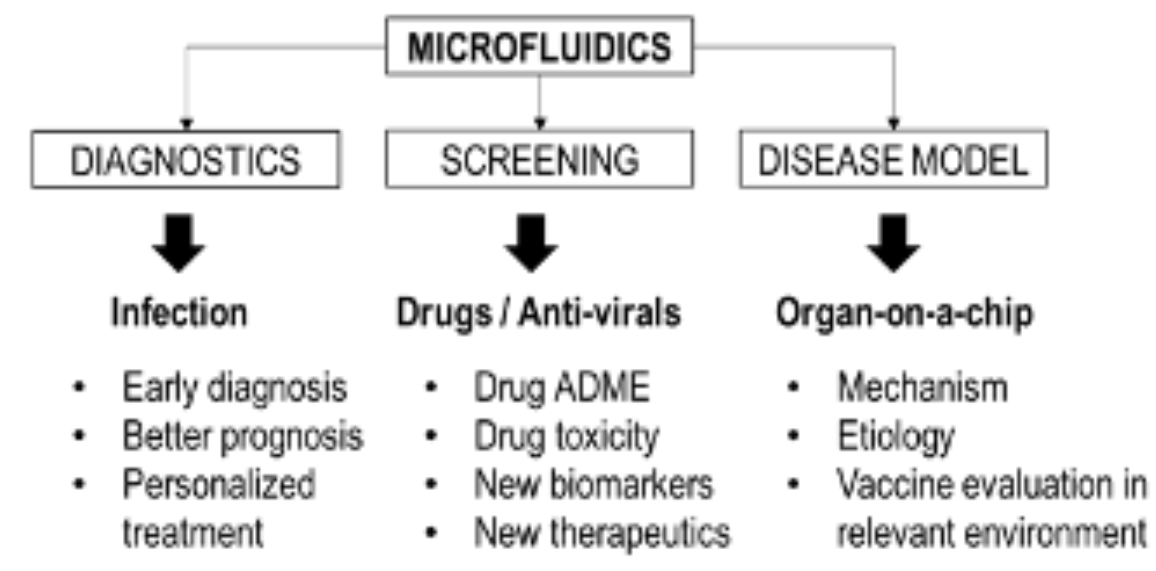

Figure 2. Contribution of microfluidics technology in virus pandemics. Three different main scenarios are identified, including diagnostics, screening, and disease modelling (ADME: administration, distribution, metabolism, and excretion).

\section{Rapid diagnosis of viral infection}

The rapid diagnosis of viral infections is of paramount importance to accelerate patient intervention and improve its prognosis. This is particularly true in viruses with high infection rates that can lead to patient death, such as COVID-19. The standard-of-actuation to diagnose (and quantify) this and other type of viruses is mainly based on real-time reverse transcription polymerase chain reaction (RT-PCR) that detects the specific genetic material of the virus. Briefly, RT-PCR uses thermal cycles to amplify the amount of DNA molecules and a specific fluorescent dye to detect the targeted genetic material. Some viruses, such as the SARS-CoV-2 only contain RNA. Therefore, to be detected by RT-PCR it is first needed to convert the RNA to DNA. This is accomplished by a process denoted as 'reverse transcription'.

The initial genetic material is gathered from samples taken from the upper respiratory tract in suspicious patients (nasopharyngeal swabs, oropharyngeal swabs, 
nasopharyngeal washes, or nasal aspirates) (Figure 3) [5]. This method is very efficient and the results can be observed while the process is ongoing. However, RT-PCR can be time consuming (it can take several days to complete), laborious (well-trained personnel is required), and it typically requires the use of bulky and expensive equipment. Other methods employed for the diagnosis of viral infection include the detection of antigens or antibodies in biological fluids by e.g., the traditional enzyme-linked immunosorbent assay (ELISA). However, this type of methods can lead to false results due to the low viral load or mutations in their genome. Considering that thousands of suspicious cases need to be efficiently tested in a daily basis, more efficient point-of-care technologies are needed for the rapid diagnosis of the viral infection, and in particular, for COVID-19.

Microfluidic chips have been widely utilized for the detection of viruses in the recent years [6, 7]. These devices typically integrate miniaturized analytical systems that can store, transport and process fluids and reagents that enable the early and reliable diagnosis of the disease-causing virus. The chips also integrate interconnected microchannels, miniaturized mixers and valves, reaction chambers and/or detectors (i.e., biosensors) to manipulate the sample and all the fluids. Interestingly, they can be combined with PCR analysis to mimic the gold-standard laboratory RT-PCR workflow but at a higher speed and efficiency, using less reagents, and at a lower cost [8]. As an example of this approach, a portable microfluidic system was recently developed for the sensitive detection of Hepatitis C, HIV, Zika, and human papilloma virus by RT-PCR [9]. This work demonstrated the feasibility to perform viral detection on-chip and the possibility to perform the analysis at the point-of-need location without sending the sample (or the patient) to the hospital. Similarly, an integrated microfluidic device was also developed for the isolation, processing, amplification, and detection of genetic viral material by RT-PCR [10]. The device prestored all the liquids and reagents needed for the reactions, which were manipulated by miniaturized pouches and valves. The chip was used to detect bacterial B. Cereus, viral armored RNA HIV, and HIV I virus in saliva. Again, the device included the RT-PCR chamber were the genetic material was amplified and detected in a fast and 
efficient manner. Finally, nasopharyngeal aspirate/swaps, microfluidic processing, and PCR analysis were combined in a point-of-care single-use microfluidic device for the rapid, low cost, and accurate diagnosis of Influenza A virus (Figure 3) [11]. The chip integrated the solid phase extraction and molecular amplification via RTPCR to amplify the RNA of the virus using human nasopharyngeal aspirate and swap directly obtained from clinical specimens. The sample preparation channel was connected directly with the RT and PCR steps. Briefly, the assay was performed first mixing the aspirate/swaps from the specimen with a lysis buffer and injected in the preconditioned channel to release the virus nucleic acids. Next, this genetic material bound to the SPE column. After several cleaning steps and the injection of reaction buffers, the extracted RNA was amplified by the RT-PCR on-chip (first, RT chamber and then PCR channel). The serpentine PCR channel was optimized to achieve repeatable and predictable temperature profiles flowing the sample through $30 \mathrm{cy}$ cles of PCR. Interestingly, this approach showed a higher sensitivity than two rapid immunoassays traditionally used, and a high specificity equivalent to the one of rapid assays and direct fluorescent antigen testing. The obtained results were validated using standard bench-top quantitative RT-PCR and similar results were obtained. Finally, the simplicity and versatility of this approach allows the system to be applied to a wide variety of virus infection and other type of pathogens.

Overall, these examples illustrate how microfluidics can accelerate the diagnostics of viruses and other pathogens. It also shows how the development of totally-integrated microfluidic systems can contribute in reducing the need to perform in situ evaluations at the hospital. This would eventually reduce the number of unnecessary visits as well as the workload in the laboratories. 


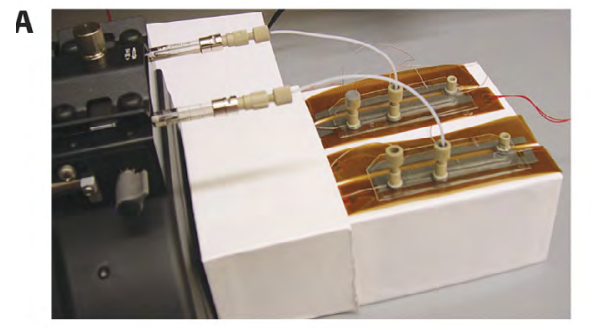

B

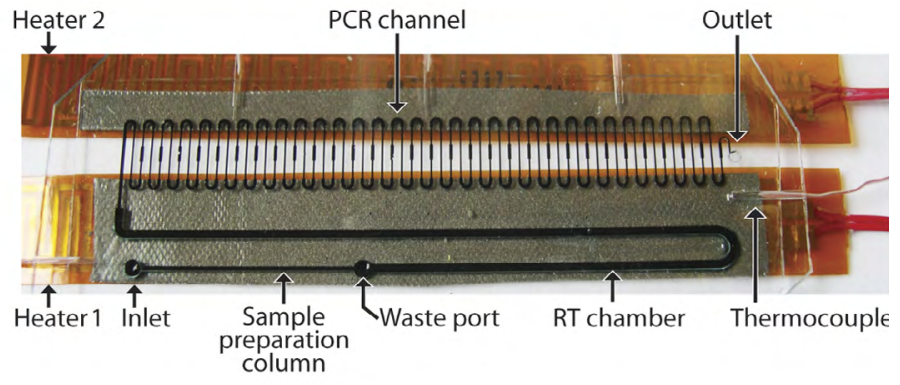

c

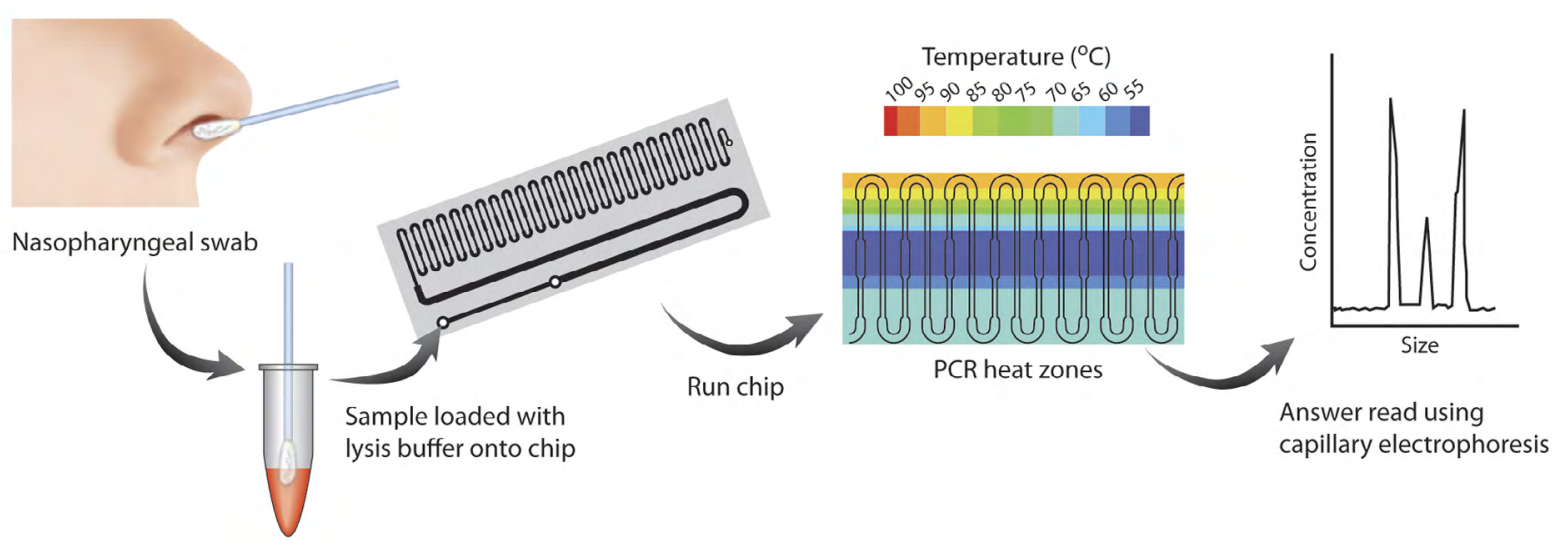

Figure 3. Microfluidic device for the rapid diagnosis of Influenza A RNA virus in human respiratory specimens. (A) Experimental set-up showing two microfluidic chips with attached thin film heaters. (B) Channel design with three sections: sample preparation, RT chamber and PCR channel. (C) Scheme showing the microfluidic assay process: the nasopharyngeal sample is mixed with lysis buffer and injected into the chip, where the PCR products are read using a commercial capillary electrophoresis system. Reproduced with permission from the publisher [11] (Creative Commons Attribution License).

\section{Drug screening and discovery}

One of the main characteristics of COVID-19 and other viruses is its genetic diversity, which generates variants of the virus with mutations. This causes different susceptibilities on patients depending on the virus characteristics and it is associated with the resistance to anti-viral drugs. The continuous discovery and screening of drugs is therefore of great importance to ensure the quality and life expectancy of the population during the current and future virus outbreak. The gold standard to investigate the efficacy of anti-viral compounds in vitro focus on using static monolayers and large populations of infected cells. However, the efficacy of a drug at a particular dosage may differ and some individual cells may respond differently. This have important implications for infection outcome and drug resistance. For this reason, it may be convenient to investigate individual infected cells. In this regard, microfluidic systems 
have an enormous potential for the screening and discovery of anti-viral drugs on individual cells due to its capability to encapsulate cells in micro-droplets. In addition, the integration of optical or electrochemical biosensors within microfluidic systems can be used to increase the sensitivity and limit of detection of key analytes related to viral infection or therapy efficacy. With this type of approach, it is possible to simultaneously observe a multitude of individual cells infected with viruses (or other pathogens), providing key insights about infection dynamics or drug efficiency. As an example, a high-throughput droplet-based microfluidic platform was employed to evaluate the efficacy of neutralizing antibodies for different variants of murine noroviruses using single virus particles incubated in a large number of pico-liter drops [12]. By identifying both sensitive and resistant viruses, this device showed the capability to estimate the potential for viral resistance to anti-viral drugs prior to their clinical use. The obtained results were validated by traditional assays, but at a lower cost and faster speed. Similarly, a microfluidic chip containing multiple micro-cavities was recently reported to assess the viral infection dynamics and inhibition on individual cells infected with enterovirus [13]. The chip was employed to investigate three classes of enterovirus inhibitors with distinct mechanisms of action. The main finding of this work was that the three compounds provided a different 'signature' related to how the virus replicated. Therefore, by comparing on-chip the signature of a compound to those of known drugs, it would be possible to narrow down the target of the drug. Importantly, and similar to the former works, this device could be applied for the discovery and screening of any class of therapeutics.

Finally, microfluidics can also significantly contribute in developing anti-viral vaccines. As an example, a recent study reported a microfluidic device to perform millions of parallelized single-virus multi droplet-based assays to screen different vaccine candidates to HIV-1. The viral particles were sorted according to the epitope expression recognized by broadly neutralizing antibodies with an impressive efficiency of $>99 \%$ [14]. Actually, the device worked as a miniaturized flow cytometer enabling fluorescence-based sorting of viral particles possessing antigenic envelope proteins. Finally, a genomic analysis could be performed on the sorted virus particles that expressed antibody-binding epitopes. Overall, this work showed how microfluidic systems could be 
used to screen in a high-throughput manner diverse virus library to reveal novel targets for virus vaccine.

To sum up, the above-mentioned demonstrates how microfluidics can be applied for the development and screening of anti-viral drugs and vaccines, showing its potential to replace, or complement, the traditional methods currently used by pharmaceutical and biotechnology companies [15]. This is particularly attractive due to their intrinsic properties, such as miniaturization and parallelization of experiments. This will univocally benefit the biomedical industry, which aims at using more clinically-relevant models but at a lower cost and faster speed. In this regard, it is worth noting that microfluidic systems are currently being utilized by regulatory agencies, such as the US Drug and Food Administration, to assess the toxicology of food supplements and additives [4]. And biotechnology and pharmaceutical companies, such as Pfizer ${ }^{\mathrm{TM}}$, Roche ${ }^{\mathrm{TM}}$, Merck ${ }^{\mathrm{TM}}$, or Johnson \& Johnson ${ }^{\mathrm{TM}}$, among others, are also employing these systems to accelerate the screening and development of novel therapeutic agents [4]. Overall, the future looks promising for microfluidics in the field of diagnosis and drug development.

\section{Disease modelling}

The combination of microfluidics, nanotechnology, biochemistry and cell biology can also be employed to elucidate the mechanisms of viral infection in key organs and tissues of the human body, thus contributing to an improved understanding on disease etiology. Among all the events to investigate, the mechanism of host-virus proteins interaction is a key factor. In the particular case of COVID-19, and as afore-mentioned, solid evidences have shown that the spike (S) viral protein of SARS-CoV-2 virus specifically binds to the ACE-2 receptor on type II lung pneumocytes to infect the cell [1]. This initial interaction triggers a cascade of critical events that affect different organs and tissues, including the lung, the kidney, the heart, and the vasculature. Therefore, its study may provide valuable insights into the biological functions of virus virulence and on the molecular basis of virus pathogenicity. In this regard, the recent advances in organ-on-a-chip technology may provide an opportunity for the development of realistic in vitro models of these organs and tissues. As an example, 
the pneumonitis induced by SARS-CoV-2 can be investigated by means of a lung-ona-chip model; the severe cardiac implications with a heart-on-a-chip; the renal damage with a kidney-on-a-chip; and the massive blood clot formation by means of a vasculature-on-a-chip. The development of these on-chip models will univocally contribute to: (i) a better understanding of the process of viral infection; (ii) the identification of new therapeutic targets and biomarkers and (iii) the evaluation of the efficacy of anti-viral pharmacological compounds. In the following, we describe several examples on the development of these four different organs-on-a-chip models, which are associated not only with COVID-19 pandemic, but also in other important viral infections.

\section{- Lung-on-a-chip}

The field of organ-on-a-chip technology emerged after the development of the lung-on-a-chip model [16]. This model reconstituted the functional alveolar-capillary interface of a human lung by seeding inside the microfluidic chip bronchiolar epithelial and microvascular endothelial cells. The chip contained two microchannels separated by a porous membrane with both cells seeded on either side mimicking the alveolar-capillary interface. Two additional lateral channels were employed to actuate on the membrane by mechanical cyclic stretching, which reproduced physiological breathing. Importantly, this model was utilized to reproduce complex integrated organ-level responses to bacteria and inflammatory cytokines introduced into the alveolar space. In this regard, this work paved the way towards applications in the field of viral infections, not only for gaining mechanistic insights, but also to evaluate the efficacy and toxicity of anti-viral compounds. Indeed, in a recent work, this model was used to reproduce the infection of the bronchiolar epithelium with $\mathrm{H} 1 \mathrm{~N} 1$ influenza $\mathrm{A}$ virus and, importantly, the emergence of drug resistance [17]. The chip was used to identify the molecular mediators of the host response to infection and to discover a potential new antiviral therapeutic that targeted these mediators. Altogether, this work showed how lung-on-a-chip technology can be applied to create a clinically-relevant model to study viral infections, representing a powerful pre-clinical tool for the development and screening of anti-viral compounds and vaccines [18]. 
Variations of this on-chip model have been also reported. As an example, a "breathing" alveolus-on-a-chip model was recently described mimicking the physiological gas exchange using primary human lung alveolar cells (Figure 4). The model reproduced the cyclic mechanical deformation produced by the diaphragm, and the airblood barrier and the air-liquid interface of the native alveoli. Interestingly, the model included both type I (ATI) and type II (ATII) pneumocytes as well as lung endothelial cells. As mentioned above, the latter cells (ATII) express the ACE-2 receptor where SARS-CoV-2 binds, and therefore, this model may be applied for the investigation of COVID-19 etiology. This model could also be used for evaluating the effect of viral infections on individuals suffering from chronic diseases, such as asthma or chronic obstructive pulmonary disease, who are considered as high risk patients [19].
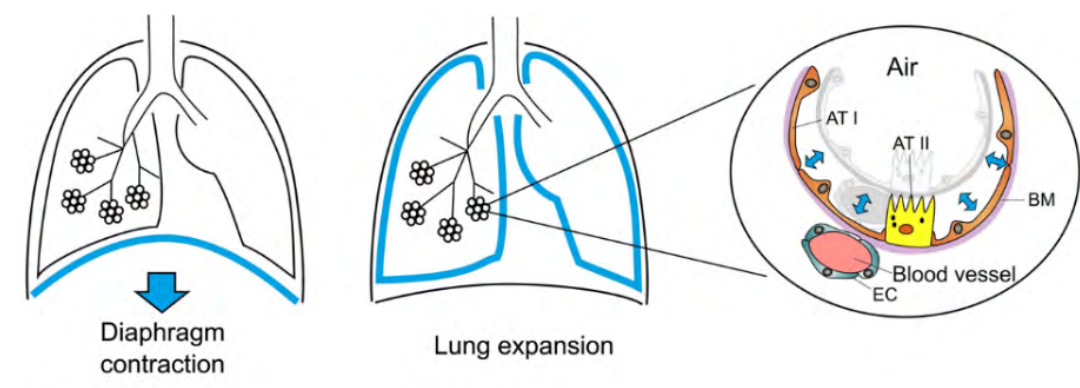

Lung expansion
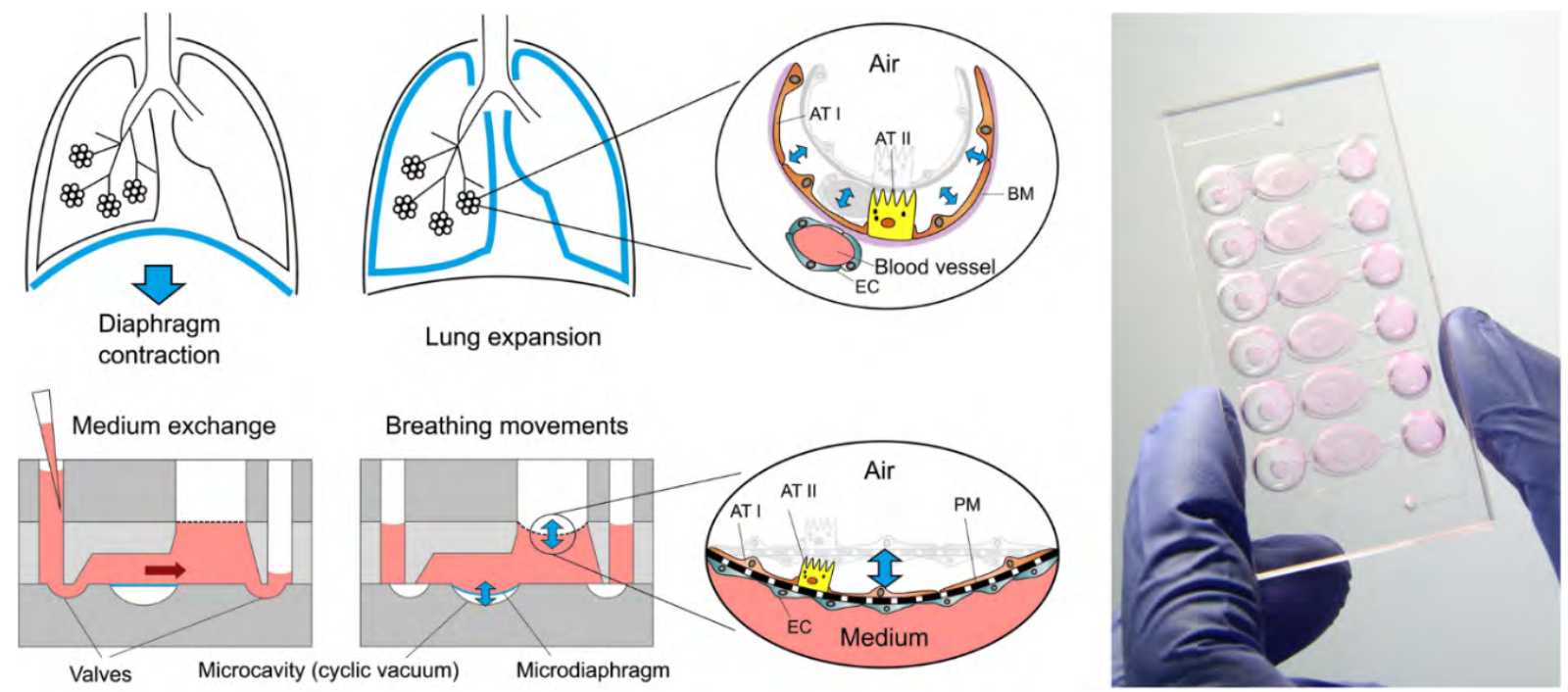

Figure 4. Lung-on-a-chip. (Top) Scheme showing the working procedure (contraction) of the lung and its functional unit - alveoli. The alveolar barrier is formed by a tight alveolar epithelial cell layer made of AT I and AT II cells together with endothelial cells (EC). (Bottom) Scheme showing the working procedure of the microfluidic alveoli model: medium exchange and breathing modes. The "breathing" is induced by applying cyclic vacuum. Two valves located on each sides are opened for medium exchange. (Right) Picture showing an array of individual lung-on-a-chip models. Reproduced with permission from the publisher [20] (Creative

Commons Attribution 4.0 International License).

\section{- Heart-on-a-chip}

Besides attacking the lungs, COVID-19 also implies severe cardiovascular complications, such as myocardial injury, myocarditis, acute myocardial infarction, heart 
failure, dysrhythmias, and venous thromboembolic events [21]. Indeed, these complications can significantly contribute to the morbidity and mortality associated with this disease [22]. A recent study showed that about 30\% of COVID-19 patients displayed myocardial injury and many other investigations have focused on the cardiovascular complications associated with COVID-19 and other viral infections [23]. The mechanism of cardiovascular impairment is not well understood. However, some evidences suggest that a widespread systemic inflammation issues resulting from the generated cytokine storm, together with the direct viral infection of the cardiovascular system, and pre-existing morbidities, may be responsible of this COVID-19-induced heart damage [24]. The excess of cytokines can indeed lead to a fatal myocarditis and the virus can directly infect cardiac cells, which also express the ACE-2 receptor. Indeed, this direct viral infection was already observed in other virus outbreaks, such as SARS-CoV [25]. Importantly, some of the drugs that were tested to treat the virus, such as hydroxychloroquine and remdesivir, showed some degree of cardiotoxicity in some patients, some of them with pre-existing issues.

The use of organ-on-a-chip models of the heart could provide multiple advantages for assessing the impact of this and other viral infections in the cardiac tissue. Heart-on-a-chip models that recapitulate the cardiac tissue level functionality and reproduce the mechanism of a human heart have been widely reported [26]. Typically, cardiomyocytes derived from human pluripotent stem cells are employed to line the chamber(s) of the chip. However, to make the cardiomyocytes contract synchronously it is important to accurately reproduce the native cellular organization of the heart by exhibiting sarcomeres assembling with aligned tissue structure. For this, heart-on-a-chip models typically incorporate microelectrodes to stimulate the cardiomyocytes to obtain a better maturation, orientation and contraction. This type of model can contribute not only to decipher human heart functions but also to investigate diseases that affect the cardiac tissue or to screen the efficacy of drugs [27]. In this regard, Agarwal et al. reported a heart-on-a-chip model for the high-throughput study of pharmacological drugs (Figure 5) [28]. This type of assay showed numerous advantages compared to pharmacological studies based on isotropic layers of cardiac 
myocytes, such as a high control over the fluid flow for injecting and washing out the drug. As a proof-of-concept, isoproterenol, a typical pharmacological agent used for the treatment of bradycardia, was injected on-chip and its effect on the contractility of electrically-stimulated cardiomyocytes investigated. The obtained results showed a dose-dependent response of the drug, which was monitored by an automatized contractility assay.

Some strategies aiming at reducing the degree of infection of COVID-19 propose the use of angiotensin II, a novel vasopressor agent recently approved in US and Europe. The rationale is that exogenous angiotensin II can bind, inhibit and down-regulate ACE-2 and, potentially, prevent SARS-CoV-2 from entering the cell [29]. However, this approach may be damaging in some other organs and tissues, such as the heart. To investigate the potential cardiac dysfunction of angiotensin II (Ang II), a heart-on-a-chip model was employed recapitulating the native laminar cardiac tissue structure [29]. To this aim, the deflections resulting from contracting healthy and Ang II-treated tissues were compared, showing that treated cardiac tissues induced pathological gene expression profiles and arrhythmia. These results showed that, even though Ang II could have some beneficial effects against the virus in the lung, it may also be cardiotoxic. Overall, this illustrates the potential of this type of organ model to identify toxicity effects of pharmacological compounds. In this regard, there is currently an intense research in the development of heart-on-a-chip systems interconnected with other organ models. Among them, the liver is an important organ to evaluate unforeseen cardiotoxic effects of drug metabolites. Some works have already shown that a drug per se is not toxic, but are their metabolites. Therefore, future organ models and drug screening applications must consider the use of advanced multi-organ-on-a-chip systems to evaluate this type of secondary toxicity. 
a
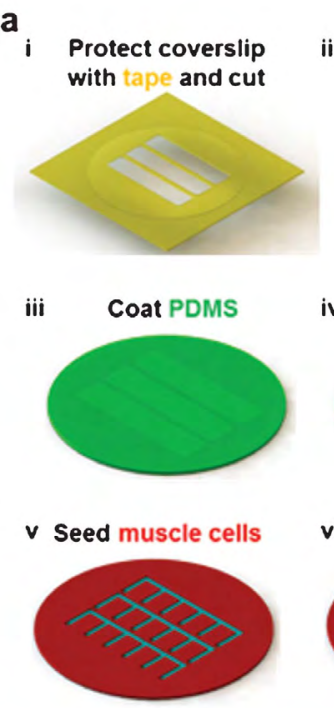

b

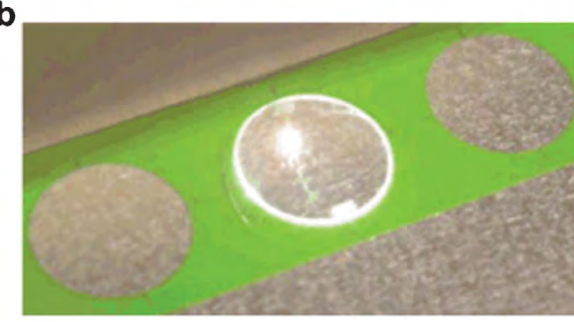

C

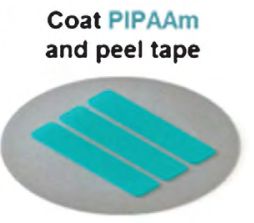

iv Cut PDMS and $\mu \mathrm{CP}$

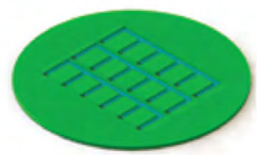

vi Record contractility

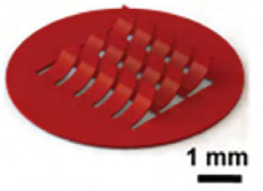

$1 \mathrm{~mm}$
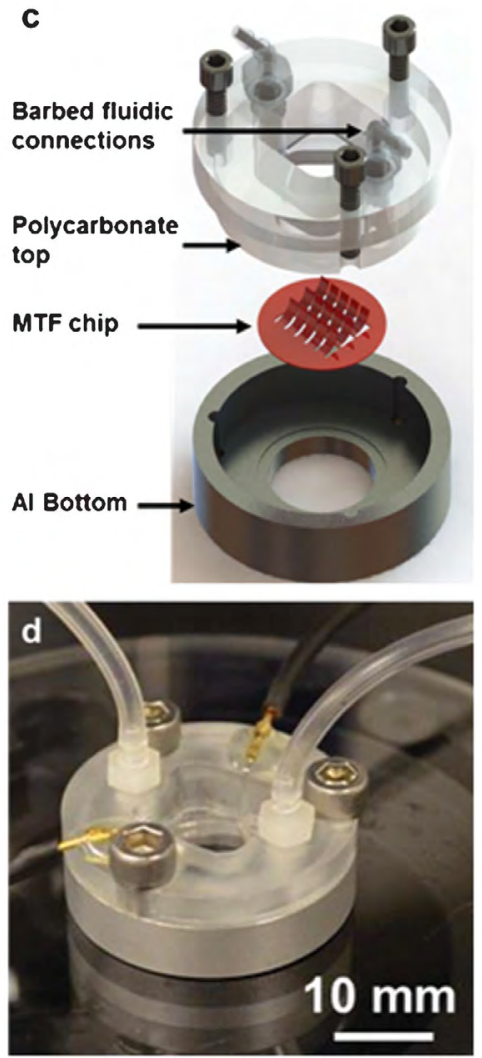

Figure 5. Heart-on-a-chip. Microfluidic device based on muscular thin films (MTF) composed of cardiomyocytes cultured on a deformable PDMS thin film. (A) Fabrication scheme; (B) Laser-induced manufacturing of the cantilevers; (C) Building blocks of the microfluidic heart-on-a-chip model; (D) Final prototype. Reprinted with permission from the publisher [28]" (Royal Society of Chemistry).

\section{- Kidney-on-a-chip}

Renal dysfunctions, such as acute kidney injury, are also related to viral infections. Many viruses, such as SARS-CoV-2, specially infect renal tubules in the kidney tissue and can be therefore a severe complication [30]. Recent evidences obtained in autopsies from deceased patients with COVID-19 showed not only a prominent acute proximal tubular injury, but also peritubular erythrocyte aggregation and glomerular fibrin thrombi with ischemic collapse, among other lesions [31]. These facts highlight that a detailed examination of kidney damage is of critical importance. Microfluidics can also help in analyzing kidney abnormalities to provide important information for future clinical interventions. As an example, a distal tubule-on-a-chip model of virus-induced kidney disease was recently developed to explore the pathogenesis of virus-related renal dysfunction using pseudorabies virus as a test bed [32]. The model 
successfully reproduced both the distal renal barrier structure of the native tissue and the sodium reabsorption function. Importantly, after viral infection, it was observed a renal dysfunction in electrolyte regulation that would eventually lead to virus-induced serum electrolyte abnormalities. Interestingly, the virus infection did not perturb the regulation function of Ang II in sodium reabsorption during the first hours after infection, thus providing key insights about the virus pathogenesis. It would be interesting to evaluate the same symptoms using SARS-CoV-2 virus due to the important role of Ang II on its pathogenesis.

Kidney-on-a-chip models have also been employed to advance in the discovery of drugs and understanding their toxicity [33]. Similar to cardiotoxicity, nephrotoxicity of anti-viral compounds is also a crucial component in drug discovery. As an example, Musah et al., recently developed human kidney glomerulus-on-a-chip model - the major site of blood filtration - using podocytes derived from human induced pluripotent stem cells recapitulating the native tissue-tissue interface of the human glomerulus in the kidney [34]. This study showed the nephrotoxicity of a common cancer drug, adriamycin, which illustrated the ability of this on-chip model to mimic the function and disease acquisition of the glomerular capillary. A similar glomerulus-on-a-chip model was also developed to mimic human hypertensive nephropathy, an important renal disease that can lead to glomerular sclerosis (scarring of the kidney blood vessels) [35]. The model reconstituted the cellular composition and functions of the native glomerulus and was used to investigate the mechanism of glomerular sclerosis caused by glomerular hypertension. The chip was formed by two perfused channels separated by a porous membrane, which was lined in the opposed layers by glomerular endothelial cells and podocytes (Figure 6). The obtained results showed that glomerular mechanical forces were fundamental in the rearrangement of the cytoskeleton as well as in cell junction damage that leads to increased glomerular leakage that is observed in hypertensive nephropathy. Overall, this model and similar ones could provide a physiologically-relevant platform for drug screening and toxicology testing, as well as for the evaluation of viral infections in the kidney.

Note finally that the effect of COVID-19 on the kidney has already been investigated using other type of models, such as animal models or organoids. In the latter, it 
was recently found that SARS-CoV-2 infection in the kidney could be prevented using a soluble ACE-2 receptor [36]. A similar approach could be therefore performed using microfluidics, which may provide additional and complementary information on the physiopathology of the disease as well as on the effects of therapeutic drugs.

A

\section{Glomerulus in vivo}

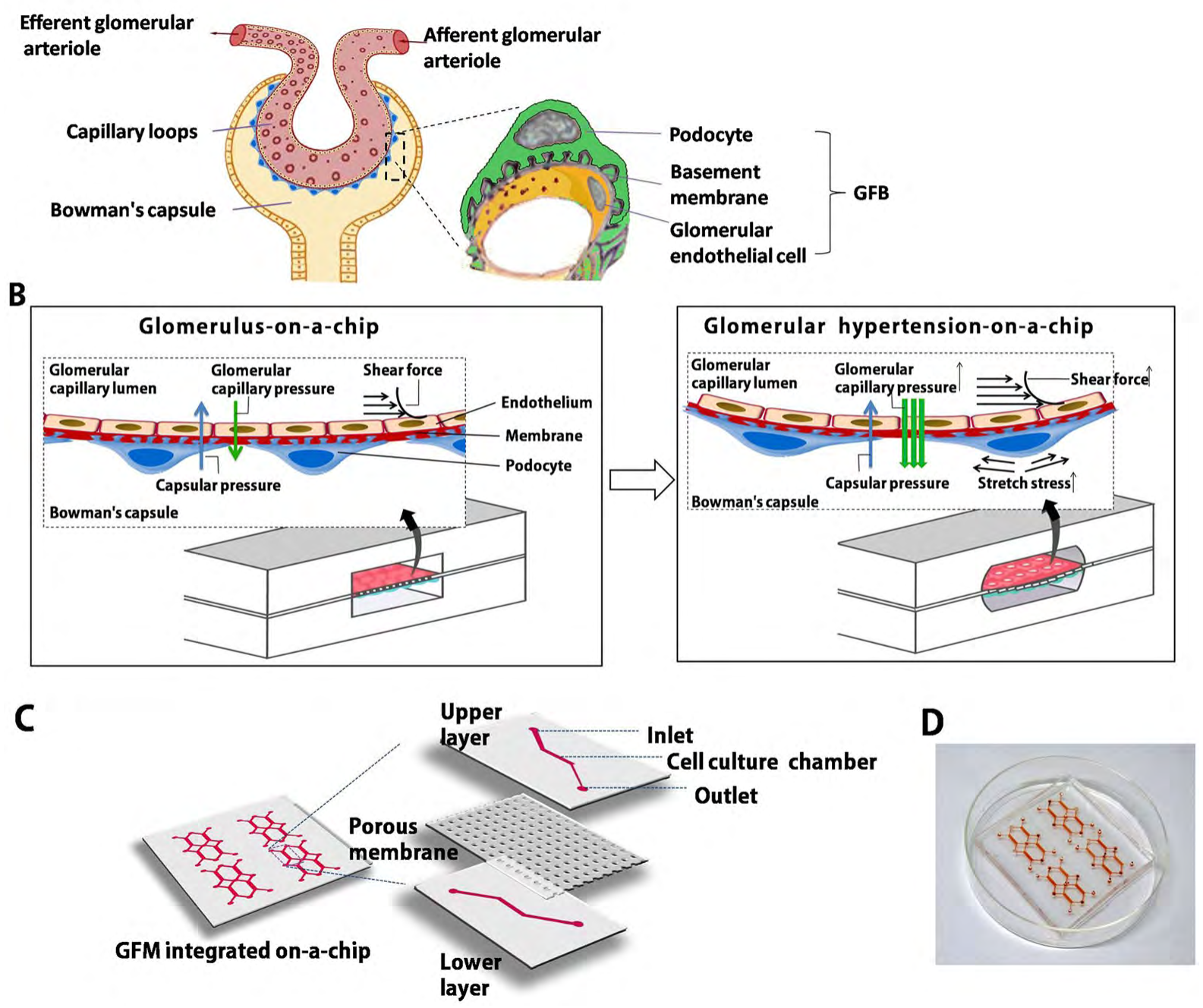

Figure 6. Kidney-on-a-chip. (A) Scheme showing the building blocks of the functional unit of the kidney - the glomerulus. In the capillary loops, the glomerular filtrate penetrates the glomerular filtration barrier (GFB) into the Bowman's - urine - capsule. (B) Schemes of the (left) healthy and (right) hypertensive glomerulus-on-a-chip and its main constituents. The model recreated the physiological glomerular filtration by perfusing the upper channel and causing mechanical forces. The modulation of the mechanical forces leads to a pathological phenotype. (C-D) Scheme and picture of an array of glomerulus-on-a-chip models. Reprinted with permission from the publisher [35]. (Creative Commons Attribution 4.0 International License). 


\section{Vasculature-on-a-chip}

Microfluidic devices and organs-on-a-chip systems can also be employed to reproduce the human vasculature, including both the blood and lymphatic vessels [37]. This type of models may find important applications in the field of nanomedicine and drug delivery, for example, by investigating the reduced target efficiency of drugs compared to the injected dose [38]. One of the main reasons behind this limited efficiency is the lack of knowledge about the behavior of drugs in the vasculature. In addition to this, other critical events occurring during viral infections justify the need of this type of vasculature models. This includes the massive formation of blood clotting occurring in COVID-19. Indeed, together with SARS-CoV-2-induced pneumonia, blood clotting is directly responsible of a large amount of deaths; and recent evidences have shown that blood clots arise in 20-30\% of COVID-19 patients [39, 40]. The mechanisms involved on this massive blood clot formation are just beginning to be investigated, but very few insights have been obtained so far. Multiple patients, including young healthy individuals, are dying from strokes caused by the blockages in the brain. Additionally, miniature clots have been also observed all around the body, including small capillaries in the lung and skin, restricting the flow of oxygenated blood [41, 42]. The current standard-of-care to treat this massive clotting is based on injecting systemically blood thinning medication (e.g., heparin or derivatives). However, these drugs are not capable to reliably prevent clotting in patients with COVID-19 or to dissolve them. In addition, a major drawback of standard anti-thrombotic medication is its toxicity, in particular, when injected for long period.

Microfluidics can contribute to gain key insights about the fundamental mechanisms of the massive clotting formation through the development of vasculature- or vessel-on-a-chip models [37]. In this regard, some microfluidic chips have already been developed to investigate the formation of blood clots (Figure 7). As an example, a serpentine-like microfluidic channel was recently developed to mimic the native capillary bed. The walls of the channel were functionalized with well-controlled concentrations of fibronectin, which promoted the aggregation of (porcine) red blood cells. This system was employed to evaluate the efficiency of clinically-used heparin and to assess the dynamics of clotting formation. However, the versatility of the chip gives 
ample space to use it for testing other blood-thinning medication, or other type of innovative compounds aiming and preventing the formation of clots or to bust them.
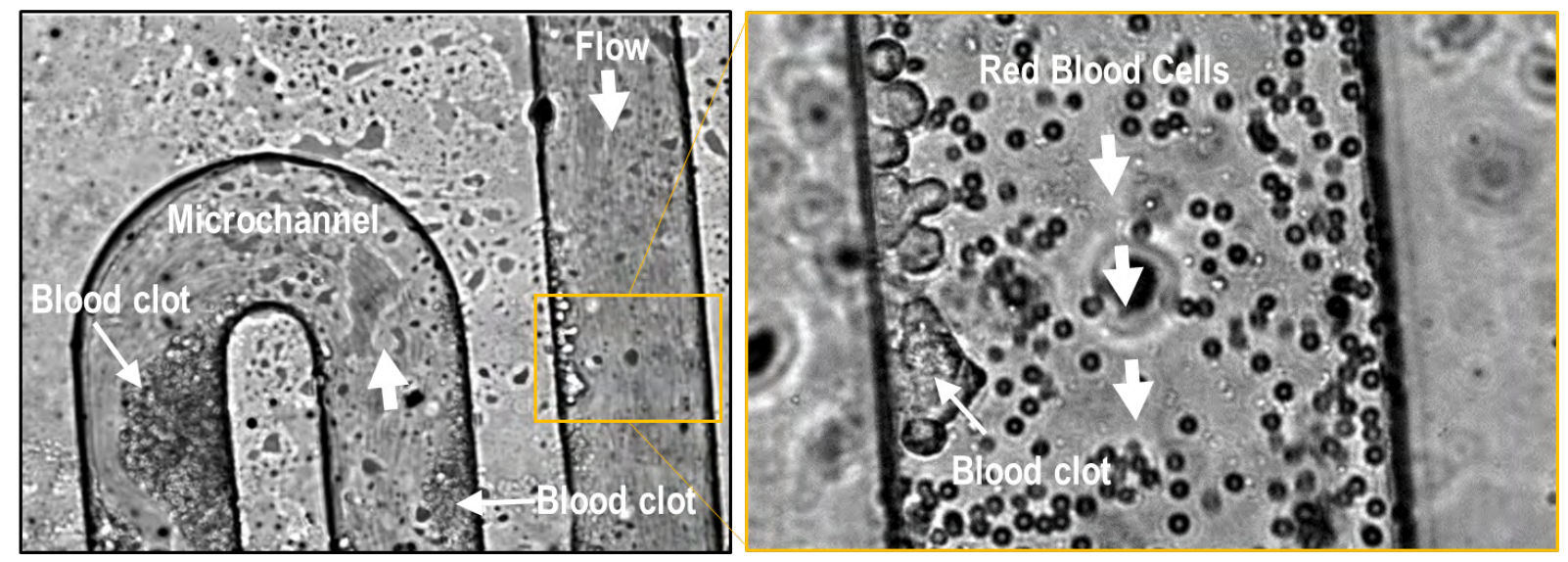

Figure 7. Vasculature-on-a-chip. (Left) Phase-contrast microscopy image of red blood cells flowing along a microfluidic channel (200 $\mathrm{mm}$ in diameter) and forming massive clotting. (Right) Detailed image of a blood clot and flow of red blood cells. Source: David Caballero.

Taken together, vasculature-on-a-chip systems, despite its simplicity, may find important applications for the study of critical events occurring during viral infection, in particular massive blood clotting, and a drug-screening platform to test the efficacy of drugs.

\section{BIOPRINTING AND VIRUS PANDEMICS}

Three-dimensional (3D) printing is considered as an innovative field in several areas, namely in tissue engineering and regenerative medicine. The $3 \mathrm{D}$ cell-printing of living tissues, called 3D bioprinting, is based on a bottom-up strategy assessing the construction of relevant tissues through the simultaneous deposition of bioinks, composed by supportive biomaterial-based matrices containing biophysical and biochemical cues, with a single or multiple mixture of living cells.

During the ongoing COVID-19 world crisis, several companies specialized in 3D printing threw helpful initiatives to restrain the widespread of the virus [43]. Their solutions and efforts have been put at the service of medical staff and patients by 
creating a myriad of $3 \mathrm{D}$ printed materials, from printed respirators, ventilators, and other medical equipment [44].

Outside the hospitals, researchers and scientists are using bioprinting technologies not only to help protect medical staff and patients but also to contribute to find a cure to this epidemic. CLECELL@, a Korean company has developed an artificial tissue and created a respiratory epithelium model earlier this year using its proprietary 3D bioprinter, the U-FABC. What is so interesting about this model is that it is expected to become a tool to test for the severe acute respiratory syndrome coronavirus (SARS-Cov-2), as well as an invaluable instrument for assessing the mechanisms of this and other viruses [45]. According to the company, since SARS-CoV-2 is less infectious towards animals, this new technology is being considered as a prospective substitute to more traditional ones (fertilized eggs to create vaccines). Instead, CLECELL's innovative bioprinting technique has the potential to become a testbed for various viruses [44].

Another out of the box example of scientists fighting COVID-19 is the case of Prellis Biologics@[45]. This bioprinting research company is studying and testing the potential of using synthetic bioprinted lymph nodes for the production of fully human antibodies for COVID-19. According to the company, the process of building the antibodies starts with bioprinting the synthetic human lymph nodes, which are posteriorly colonized with heal thy human blood cells. The synthetic lymph nodes are then "vaccinated" with promising formulations after being exposed to the virus. In this complex process, the cells that produce the antibodies divide from non-responding cells, making it possible to isolate them. From there, antibody sequences can be produced in therapeutic quantities in collaboration with biotech or pharmaceutical companies [45].

Many studies already published, namely in the field of 3D bioprinting in the area of lung and immune systems, could be potentially applicable in the future, both for studying the pathology of COVID-19 and accelerate the drug discovery process. However, the real potential to create models for viral infection studies has not yet been fully realized [46, 47]. In a study conducted by Berg et al. [46], it was described the optimization of a bioink composition for extrusion printing and a $3 \mathrm{D}$ model for the infection of Influenza A was achieved. A hydrogel mix of alginate, gelatin and Matrigel 
was used as a scaffold for the 3D arrangement of A549 human alveolar cells [46]. Infection of the 3D model with a seasonal Influenzas' A strain resulted in widespread distribution of the virus and also a typical clustered infection, which does not happen in two-dimensional cell culture. The bioink supported viral replication and pro-inflammatory interferon release of the infected cells, therefore exhibiting a basic immune response by releasing the antiviral IL-29 (interferon $\lambda 1$ ) [46].

Overall, these examples show how scientists can contribute in the field of bioprinted in vitro models of disease in order to develop a technological platform to investigate and fight COVID-19 and other future virus pandemics.

\section{CONCLUSION}

During the last decades, different viral outbreaks have affected the population worldwide causing a large amount of deaths, including, among others, the MERS, Ebola, Zika, SARS-CoV, and more recently SARS-CoV-2. The recent history has demonstrated the need for innovative technological solutions capable of investigating the mechanism of action of these pathogens, or to evaluate in a fast and cost-effective manner the efficacy of anti-viral drugs for future viral outbreaks. In this regard, microfluidic systems have demonstrated to be a valuable tool for the detailed investigation of viral infection and its treatment. Microfluidic analytical systems, together with lab- and organ-on-a-chip platforms can be employed to gain mechanistic insights about disease etiology. They can also be employed for the early diagnosis of the disease at point-ofneed locations and to screen the efficiency of drugs in native-like conditions. Overall, the applications of microfluidics in viral cell biology are vast, and may be considered as a promising clinical tool to complement current analytical technologies for the rapid diagnostics of pathologies, as well as for drug discovery and drug screening. Moreover, the future of translational human immunology is bright in many research fields and, in particular, in 3D bioprinting. Continuing to develop more sophisticated models of human immunity will improve our chances of successfully translating findings from fundamental biological studies to the clinic. A main goal should be to continue to develop tools to close the animal gap, to develop human-based systems that have the 
advantages of animal models, including high throughput and the potential for mechanistic, well-controlled studies. Strong collaborations between scientists, engineers, and clinicians will help to accelerate this process.

Finally, it is worth mentioning that at the 3B's Research Group, I3Bs from University of Minho, we are developing a new generation of organ-on-a-chip models for the study of physiopathological events and innovative bioprinted in vitro models of disease. We are convinced that such models will find vast applications in the clinics and biomedical industry in the near future due to their advanced capabilities.

\section{ACKNOWLEDGEMENTS}

D. Caballero acknowledges the financial support from the Portuguese Foundation for Science and Technology under the program CEEC Individual 2017 (CEECIND/00352/2017) and the project 2MATCH (02/SAICT/2017 - no 028070) funded by the Programa Operacional Regional do Norte supported by FEDER. M. Carvalho would like to acknowledge IET Harvey Research Prize 2017. The authors also acknowledge the financial support from the EU Framework Programme for Research and Innovation Horizon 2020 on Forefront Research in 3D Disease Cancer Models as in vitro Screening Technologies (FoReCaST- no. 668983), the Portuguese Foundation for Science and Technology (FCT) distinction attributed to J. M. Oliveira (IF/00423/2012, IF/01285/2015) and FCT, Fundo Europeu de Desenvolvimento Regional (FEDER) and Programa Operacional Competitividade e Internacionalização (POCI) for funding the projects B-Liver (PTDC/EMD-EMD/29139/2017), Hierarchitech (M-ERA-NET/0001/2014) and 3BioMeD (JICAM/0001/2017). 


\section{REFERENCES}

[1] Hoffmann, M., Kleine-Weber, H., Schroeder, S., Krüger, N. Herrler, T. , Erichsen, S., Schiergens, T.S., Herrler, G., Wu, N.-H., Nitsche, A., Müller, M.A., Drosten, C. \& Pöhlmann, S. (2020). SARS-CoV-2 Cell Entry Depends on ACE2 and TMPRSS2 and Is Blocked by a Clinically Proven Protease Inhibitor. Cell, 181(2), 271-280.e278.

[2] Seyhan, A.A. (2019). Lost in translation: the valley of death across preclinical and clinical divide - identification of problems and overcoming obstacles Transl Med Commun, 4, 18.

[3] Whitesides, G.M. (2006). The origins and the future of microfluidics.Nature, 442(7101), 368-373.

[4] Caballero, D., Kaushik ,S.,. Correlo, V.M, Oliveira, J.M., Reis, R.L. \& Kundu, S.C. (2017). Organ-on-chip models of cancer metastasis for future personalized medicine: from chip to the patient. Biomaterials, 149, 98-115.

[5] Udugama, B., Kadhiresan, P., Kozlowski, H.N., Malekjahani, A., Osborne, M., Li, V.Y.C., Chen, H., Mubareka, S., Gubbay, J.B. \& Chan, W.C.W.(2020). Diagnosing COVID-19: The Disease and Tools for Detection. ACS Nano, 14 (4), 3822-3835.

[6] Zhu, H., Fohlerová, Z., Pekárek, J., Basova \& E. Neužil, P. (2020). Recent Advances in Lab-On-A-Chip Technologies for Viral Diagnosis. Biosens Bioelectron, 153, 112041-112041.

[7] Simpson, C., Lee, S.S. , Lee, C.-S. \& Yamauchi, Y. (2018). Microfluidics: an Untapped Resource in Viral Diagnostics and Viral Cell Biology. Curr Clin Microbiol Rep, 5, 245-251.

[8] Chang, C.M., Chang, W.H., Wang, C.H., Wang, J.H., Mai, J.D. \& Lee, G.B. (2013). Nucleic acid amplification using microfluidic systems. Lab Chip, 13, 1225-1242.

[9] Powell, L., Wiederkehr, R.S., Damascus, P., Fauvart, M., Buja, F., Stakenborg, T., Ray, S.C., Fiorini, P. \& Osburn, W.O.(2018). Rapid and sensitive detection of viral nucleic acids using silicon microchip. Analyst, 143 (11), 2596-2603.

[10] Chen, D., Mauk, M., Qiu, X., Liu, C., Kim, J., Ramprasad, S., Ongagna, S., Abrams, W.R., Malamud, D., Corstjens, P.L.A.M. \& Bau, H.H. (2010). An Integrated, Self-Contained Microfluidic Cassette for Isolation, Amplification, and Detection of Nucleic Acids. Biomed Microdevices, 12(4), 705-719.

[11] Cao, Q., Mahalanabis, M., Chang, J., Carey, B. , Hsieh, C., Stanley, A.,. Odell, C.A, Mitchell, P., Feldman, J., Pollock \& N.R. Klapperich, C.M. (2012). Microfluidic Chip for Molecular Amplification of Influenza A RNA in Human Respiratory Specimens. PLoS One, 7(3), e33176.

[12] Tao, Y., Rotem, A., Zhang, H., Chang, C.B., Basu, A., Kolawole, A.O., Koehler, S.A., Ren, Y., Lin, J.S., Pipas, J.M., Feldman, A.B., Wobus, C.E. \& Weitz, D.A. (2015). Rapid, targeted and culture-free viral infectivity assay in drop-based microfluidics. Lab Chip, 15 (19), 3934-3940.

[13] Liu, W., Caglar, M.U., Mao, Z., Woodman, A., Arnold, J.J., Wilke, C.O. \& Cameron, C.E. (2019). More than efficacy revealed by single-cell analysis of antiviral therapeutics. Sci Adv, 5(10), eaax4761. 
[14] Chaipan, C., Pryszlak, A., Dean, H., Poignard, P., Benes, V., Griffiths, A.D. \& Merten, C.A. (2017). Single-Virus Droplet Microfluidics for High-Throughput Screening of Neutralizing Epitopes on HIV Particles. Cell Chem Biol, 24, 751-757.e753.

[15] Chi, C.-W., Ahmed, A.R., Dereli-Korkut, Z. \& Wang, S. (2016). Microfluidic cell chips for high-throughput drug screening. Bioanalysis, 8 (9), 921-937.

[16] Huh, D., Matthews, B.D., Mammoto, A., Montoya-Zavala, M., Hsin, H.Y. \& Ingber, D.E. (2010). Reconstituting Organ-Level Lung Functions on a Chip. Science, 328(5986), 1662-1668.

[17] Si, L., Prantil-Baun, R., Benam, K.H., Bai, H., Rodas, M., Burt, M. \& Ingber, D.E. (2019). Discovery of influenza drug resistance mutations and host therapeutic targets using a human airway chip. bioRxiv, 685552 .

[18] Huh, D., Leslie, D.C., Matthews, B.D., Fraser, J.P., Jurek, S., Hamilton, G.A., Thorneloe, K.S., McAlexander, M.A. \& Ingber, D.E. (2012). A Human Disease Model of Drug Toxicity-Induced Pulmonary Edema in a Lung-on-a-Chip Microdevice. Sci Transl Med, 4(159), 159ra147.

[19] Benam, K.H., Villenave, R., Lucchesi, C., Varone, A., Hubeau, C., Lee, H.-H., Alves, S.E., Salmon, M., Ferrante, T.C., Weaver, J.C., Bahinski, A., Hamilton, G.A. \& Ingber, D.E. (2016). Small Airway-On-A-Chip Enables Analysis of Human Lung Inflammation and Drug Responses in Vitro. Nat Methods, 13(2), 151-157.

[20] Stucki, J.D., Hobi, N., Galimov, A., Stucki, A.O., Schneider-Daum, N., Lehr, C.-M., Huwer, H., Frick, M., Funke-Chambour, M., Geiser, T. \& Guenat, O.T. (2018). Medium Throughput Breathing Human Primary Cell Alveolus-On-Chip Model. Sci Rep, 8(1), 14359.

[21] Long, B., Brady, W.J., Koyfman, A. \& Gottlieb, M. (2020). In Reply to MS 22060-Diagnosis of Extrapulmonary Tuberculosis and Avoiding Anchoring Bias. Am JEmerg Med, S0735-6757(20), 30030-30039.

[22] Huang, C., Wang, Y., Li, X., Ren, L., Zhao, J., Hu, Y., Zhang, L., Fan, G., Xu, J., Gu, X., Cheng, Z., Yu, T., Xia, J., Wei, Y., Wu, W., Xie, X., Yin, W., Li, H. , Liu, M., Xiao, Y., Gao, H., Guo, L., Xie, J., Wang, G., Jiang, R., Gao, Z., Jin, Q., Wang J. \& Cao, B. (2020). Clinical features of patients infected with 2019 novel coronavirus in Wuhan, China. Lancet, 395, 497-506.

[23] Guo, T., Fan, Y., Chen, M., Wu, X., Zhang, L., He, T., Wang, H., Wan, J., Wang, X. \& Lu, Z. (2020). Cardiovascular Implications of Fatal Outcomes of Patients With Coronavirus Disease 2019 (COVID-19). JAMA Cardiol, https://doi.org/10.1001/jamacardio.2020.1017.

[24] Wang, L., Zhang, Y. \& Zhang, S. (2020). Cardiovascular Impairment in COVID-19: Learning From Current Options for Cardiovascular Anti-Inflammatory Therapy. Front Cardiov Med, 7, 78.

[25] Yu, C.M., Wong, R.S.M., Wu, E.B., Kong, S.L,. Wong, J., Yip, G.W.K., Soo, Y.O.Y., Chiu, M.L.S., Chan, Y.S., Hui, D., Lee, N., Wu, A., Leung, C.B. \& Sung, J.J.Y.(2006). Cardiovascular complications of severe acute respiratory syndrome. Postgrad Med J, 82(964), 140-144.

[26] Kitsara, M., Kontziampasis, D., Agbulut, O. \& Chen, Y. (2019). Heart on a chip: Micro-nanofabrication and microfluidics steering the future of cardiac tissue engineering. Microelectronic Engineering, 203-204, 44-62. 
[27] Zhao, Y., Rafatian, N., Wang, E.Y., Wu, Q., Lai, B.F.L., Lu, R.X., Savoji, H. \& Radisic, M. (2020). Towards chamber specific heart-on-a-chip for drug testing applications. Adv Drug Deliv Rev, https://doi. org/10.1016/j.addr.2019.12.002.

[28] Agarwal, A., Goss, J.A., Cho, A., McCain, M.L. \& Parker, K.K. (2013). Microfluidic heart on a chip for higher throughput pharmacological studies. Lab on a Chip, 13(18), 3599-3608.

[29] Busse, L.W., Chow, J.H., McCurdy, M.T. \& Khanna, A.K. (2020). COVID-19 and the RAAS-a Potential Role for Angiotensin II?. Crit Care, 24(1), 136.

[30] Batlle, D., Soler, M.J., Sparks, M.A., Hiremath, S., South, A.M., Welling, P.A. \& Swaminathan, S.( 2020). Acute Kidney Injury in COVID-19: Emerging Evidence of a Distinct Pathophysiology. J Am Soc Nephrol, ASN.2020040419.

[31] Su, H., Yang, M., Wan, C., Yi, L.-X., Tang, F., Zhu, H.-Y., Yi, F., Yang, H.-C., Fogo, A.B., Nie, X. \& Zhang, C. (2020). Renal histopathological analysis of 26 postmortem findings of patients with COVID-19 in China. Kidney Internatl, https://doi.org/10.1016/j.kint.2020.04.003.

[32] Wang, J., Wang, C., Xu, N., Liu, Z.F., Pang, D.W. \& Zhang, Z.L. (2019). A Virus-Induced Kidney Disease Model Based on Organ-On-A-Chip: Pathogenesis Exploration of Virus-Related Renal Dysfunctions. Biomaterials, 219, 119367.

[33] Paoli, R. \& Samitier, J. (2016). Mimicking the Kidney: A Key Role in Organ-on-Chip Development. Micromachines, 7(7), 126.

[34] Musah, S., Mammoto, A., Ferrante, T.C., Jeanty, S.S.F., Hirano-Kobayashi, M., Mammoto, T., Roberts, K. Chung, S., Novak, R., Ingram, M., Fatanat-Didar, T., Koshy, S., Weaver, J.C., Church, G.M. \& Ingber, D.E. (2017). Mature Induced-Pluripotent-Stem-Cell-Derived Human Podocytes Reconstitute Kidney Glomerular-Capillary-Wall Function on a Chip. Nat Biomed Eng, 1, 0069.

[35] Zhou, M., Zhang, X., Wen, X., Wu, T., Wang, W., Yang, M., Wang, J., Fang, M., Lin B. \& Lin, H. (2016). Development of a Functional Glomerulus at the Organ Level on a Chip to Mimic Hypertensive Nephropathy. Sci Rep, 6, 31771.

[36] Monteil, V., Kwon, H., Prado, P., Hagelkrüys, A., Wimmer, R.A., Stahl, M., Leopoldi, A., Garreta, E., Hurtado del Pozo, C., Prosper, F., Romero, J.P., Wirnsberger, G., Zhang, H., Slutsky, A.S., Conder, R., Montserrat, N., Mirazimi, A. \& Penninger, J.M. (2020). Inhibition of SARS-CoV-2 Infections in Engineered Human Tissues Using Clinical-Grade Soluble Human ACE2. Cell, 181(4), 905-913.e7.

[37] Luque-González, M.A., Reis, R.L., Kundu, S.C. \& Caballero, D. (2020). Human Microcirculation-onChip Models in Cancer Research: Key Integration of Lymphatic and Blood Vasculatures. Advanced Biosystems, https://doi.org/10.1002/adbi.202000045.

[38] Caballero, D., Blackburn, S.M., de Pablo, M., Samitier, J. \& Albertazzi, L. (2017). Tumour-vessel-on-achip Models for Drug Delivery. Lab Chip, 17(22), 3760-3771. 
[39] Poissy, J., Goutay, J., Caplan, M., Parmentier E., Duburcq, T., Lassalle, F., Jeanpierre, E., Rauch, A., Labreuche, J. \& Susen, S. (2020). Pulmonary Embolism in COVID-19 Patients: Awareness of an Increased Prevalence. Circulation, https://doi.org/10.1161/CIRCULATIONAHA.120.047430.

[40] Klok, F.A., Kruip, M.J.H.A., van der Meer, N.J.M., Arbous, M.S., Gommers, D.A.M.P.J., Kant, K.M., Kaptein, F.H.J., van Paassen, J., Stals, M.A.M., Huisman, M.V. \& Endeman, H.(2020). Incidence of thrombotic complications in critically ill ICU patients with COVID-19.Thromb Res, 191, 145-147.

[41] Magro, C., Mulvey, J.J., Berlin, D., Nuovo, G., Salvatore, S., Harp, J., Baxter-Stoltzfus, A. \& Laurence, J. (2020). Neuroimaging Findings in Patients with COVID-19. Transl Res, S1931-5244(1920), 30070-30070.

[42] Fogarty, H., Townsend, L., Ni Cheallaigh, C., Bergin, C., Martin-Loeches, I., Browne, P., Bacon, C.L., Gaule, R., Gillett, A., Byrne, M., Ryan, K., O’Connell, N., O’Sullivan, J.M., Conlo, N. \&, O’Donnell, J.S. (2020). COVID-19 Coagulopathy in Caucasian Patients. Br J Haematol, 189(6), 1044-1049.

[43] Zhou, P., Huang, Z., Xiao, Y., Huang, X. \& Fan XG. (2020). Protecting Chinese healthcare workers while combating the 2019 novel coronavirus. Infect Control Hosp Epidemiol, 41(6), 745-746.

[44] Print, D. CLECELL: A New Bioprinted Model Could Aid COVID--19 Vaccine Testing. Available from: https://3dprint.com/266306/clecell-a-new-bioprinted-model-could-aid-COVID-19-vaccine-testing/.

[45] Network, D.P.M. Prellis using bioprinted lymph nodes to develop antibodies to SARS-CoV-2. 2020 [cited 2020 03/06/2020]; Available from: https://www.3dprintingmedia.network/prellis-bioprinted-lymph-nodes-COVID-antibodies/.

[46] Berg, J., Hiller, T., Kissner, M. S.,Qazi,T.H., Duda, G. N., Hocke, A. C., Hippenstiel, S., Elomaa, L., Weinhart, M., Fahrenson, C. \& Kurreck, J. (2018). Optimization of cell-laden bioinks for 3D bioprinting and efficient infection with influenza A virus. Sci Rep, 8(1), 13877.

[47] Murphy, S.V., De Coppi, P. \& Atala, A. (2020). Opportunities and challenges of translational 3D bioprinting. Nat Biomed Eng, 4(4), 370-380. 